\title{
Low-Cost Reusable Instrumentation for Laparoendoscopic Single-Site Nephrectomy: Assessment in a Porcine Model
}

\author{
Riccardo Autorino, M.D., Ph.D., ${ }^{1,2}$ Fernando J. Kim, M.D., ${ }^{3}$ Abhay Rane, M.D., ${ }^{4}$ Marco De Sio, M.D., Ph.D., ${ }^{2}$ \\ Robert J. Stein, M.D., Rocco Damiano, M.D., Salvatore Micali, M.D., J Jorge Correia-Pinto, M.D., Ph.D., \\ Jihad H. Kaouk, M.D., ${ }^{1}$ and Estevão Lima, M.D., Ph.D. ${ }^{7}$
}

\begin{abstract}
Purpose: To test different sets of prebent instruments and a new reusable access device for laparoendoscopic single-site (LESS) surgery.

Materials and Methods: Three surgeons with previous experience in LESS performed 12 nephrectomies in six pigs. In all procedures, a multichannel access device $\left(\mathrm{X}-\mathrm{CONE}{ }^{\mathrm{TM}}\right)$ and a 5-mm extra-long telescope were used. Four sets of prebent instruments with different profiles (S-portal ${ }^{\mathrm{TM}}$ ) were tested: Standard (one straight scissors and one curved grasper), Cuschieri, Carus, and Leroy set (each of them consisting of two curved instruments with different configurations). Assessment was performed based on both objective (procedure time; time to manage the pedicle; time to free kidney) and subjective parameters (entry/exit of instruments; triangulation; dissection up/down; dissection lateral; retraction; interdependence). The subjective assessment tool used was a Likert type scale $(1=$ easy to $5=$ prohibitive). The access device was assessed by using objective (time to complete insertion of device after skin incision) and subjective (significant air leakage, movement constraint) parameters.

Results: Time to insertion of the X-CONE was $<1$ minute in all the cases. Surgeons reported significant insufflant leakage in $58 \%$ of cases. The procedure was completed in 10/12 (83\%) cases. Mean operative time was $8.3 \pm 4.2$ minutes, being lower for the Carus group $(4.5 \mathrm{~min})$ and higher for the standard group (13 min). Among the different sets, the standard one obtained the best mean scores for all subjective parameters.

Conclusions: X-CONE allows easy abdominal access, and its reusable properties represent cost savings for LESS compared with disposable devices. Prebent instruments might also represent attractive low-cost tools for LESS.
\end{abstract}

\section{Introduction}

E FFORTS TO FURTHER REDUCE the morbidity and improvie the cosmetic outcome of laparoscopic surgery have led to the evolution of a novel surgical approach, now collectively known as laparoendoscopic single-site (LESS) surgery. ${ }^{1}$

LESS access can be obtained either by performing a single skin and fascial incision through which a single multichannel access platform is placed (single-port) or by placing several low profile ports through separate fascial incisions (singlesite). Rane and associates ${ }^{2}$ first reported the urologic use of a single multifunctional port, while the first multitrocar single- incision transumbilical nephrectomy was reported by Raman and colleagues. ${ }^{3}$ Since then, several other clinical series have been reported, and the entire spectrum of urologic procedures both for upper and lower urinary tract diseases has been described and shown to be feasible. ${ }^{4,5}$

Despite representing an evolution of standard laparoscopy, LESS surgery defies the most basic laparoscopic concepts, including triangulation of working instruments and external spacing to decrease clashing. Thus, the transition from standard laparoscopy to LESS surgery has represented a major paradigm shift. To overcome technical limitations related to LESS surgery, industries have also developed a new

\footnotetext{
${ }^{1}$ Glickman Urological and Kidney Institute, Cleveland Clinic, Cleveland, Ohio.

${ }^{2}$ Urology Clinic, Second University of Naples, Naples, Italy.

${ }^{3}$ Division of Urology, Denver Health Medical Center and University of Colorado Health Sciences Center, Denver, Colorado.

${ }^{4}$ Department of Urology, East Surrey Hospital, Redhill, United Kingdom.

${ }^{5}$ Department of Urology, Magna Graecia University, Catanzaro, Italy.

${ }^{6}$ Department of Urology, University of Modena \& Reggio Emilia, Modena, Italy.

${ }^{7}$ Life and Health Sciences Research Institute, School of Health Sciences, University of Minho, Braga, Portugal.
} 
generation of purpose-built optics and instruments. ${ }^{6}$ Articulating instruments have been developed conceptually to allow surgeons' hands to be positioned farther apart while some triangulation is created internally.

A recognized issue that is related to these new devices is cost. In general, the use of disposable instruments increases the cost of laparoscopic procedures, and the same is likely to happen for LESS surgery.

The aims of this study were to describe and assess a new reusable access device for single-port laparoscopy and to assess the feasibility of performing LESS nephrectomy in the porcine model by using four different sets of prebent, reusable instruments that were designed originally for gallbladder removal.

\section{Materials and Methods}

\section{Study design}

Nephrectomies were performed bilaterally in a porcine model by three experienced laparoscopic surgeons (FK, JHK, AR) with previous clinical experience (more than 30 cases performed) in LESS kidney surgery.

Six domestic pigs that weighed between 20 and $25 \mathrm{~kg}$ were included in the experiment, and the protocol was approved by the Animal Care Committee of Life and Health Sciences Research Institute, School of Health Sciences, University of Minho, Braga. All animals were allowed a minimum period of 72 hours before the procedure to recover from the stress of transportation. Food was withheld for 12 hours before anesthesia. All procedures were performed under general anesthesia, with 6.0-mm endotracheal intubation (Super Safety clear tracheal tube, Ruschelit $\left.{ }^{\circledR}\right)$ and mechanical ventilation. Preanesthesia medication consisted of an intramuscular injection of $32 \mathrm{mg} / \mathrm{mL}$ azaperone that was reconstituted with $1 \mathrm{mg} / \mathrm{mL}$ midazolam at a dose of 0.15 to $0.2 \mathrm{~mL} / \mathrm{kg}$. Anesthesia was induced with $3 \mu \mathrm{g} / \mathrm{kg}$ fentanyl, $10 \mathrm{mg} / \mathrm{kg}$ thiopental sodium, and $1 \mathrm{mg} / \mathrm{kg}$ vecuronium. For infection prophylaxis, all animals received an intramuscular injection of $1 \mathrm{~g}$ ceftriaxone before endoscopy. Anesthesia was maintained with $1.5 \%$ to $2 \%$ sevoflurane and a perfusion of $1 \mathrm{mg} / \mathrm{kg} / \mathrm{h}$ vecuronium. High doses of anesthesia were administered for euthanasia.

In each pig, nephrectomies were performed bilaterally. Each procedure was accomplished by following the same surgical steps: Port placement, dissection of the renal pedicle, clipping and division of the renal pedicle, and freeing of the whole kidney.

Each surgeon (FK, JHK, AR) performed four nephrectomies, one with each of the four different instrument sets. Overall, 12 nephrectomies (6 right and 6 left) were performed.

\section{Instruments}

For all the procedures a multichannel access device (X-CONE, ${ }^{\text {TM }}$ Karl Storz, Tuttlingen, Germany) was used (Fig. 1). This system consists of a cone (size $25 \mathrm{~mm}$ ) comprising two half cones with groove and tongue; a multivalve sealing cap with $4 \times 5 \mathrm{~mm}$ and $1 \times 5-13 \mathrm{~mm}$ channels; and an insufflation stopcock with Luer-Lock connector.

To insert the device, a minilaparotomy is performed (approximately $1 \mathrm{~cm}$ long incision in the pig). Afterward, the atraumatic $\mathrm{X}$-CONE halves are successively inserted in a similar manner as retractors and joined to form a sealing cone using a pivoting movement. Then, the seal is snapped on.

A 5-mm Hopkins II extended length $(50 \mathrm{~cm}) 5 \mathrm{~mm} \mathrm{30-}$ degree scope (Karl Storz, Tuttlingen, Germany) was used in all cases.

The following sets of prebent instruments (S-portal series, Karl Storz, Tuttlingen, Germany) were used (Fig. 2):
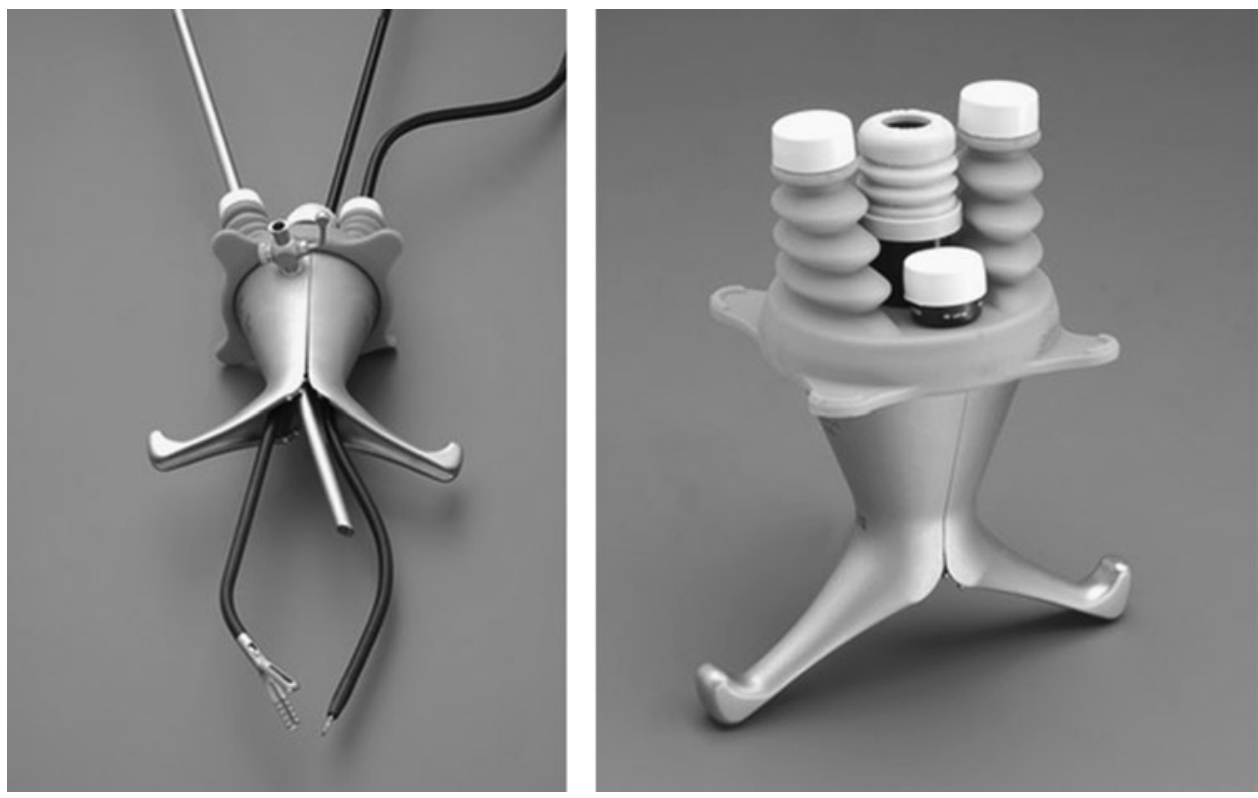

FIG. 1. X-CONE ${ }^{\mathrm{TM}}$ reusable single-port platform. 


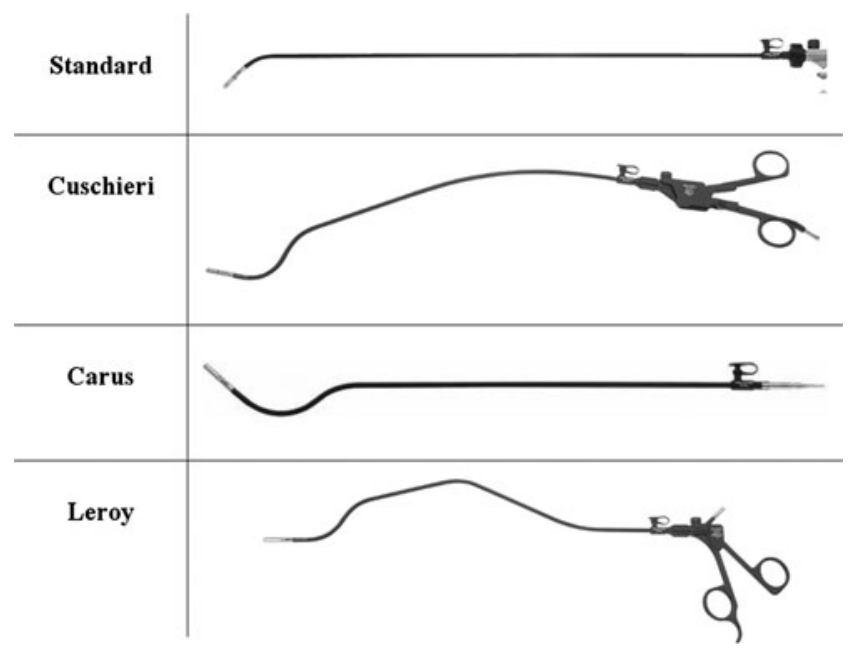

FIG. 2. Prebent instruments for laparoendoscopic singlesite surgery.

- Standard set, consisting of one straight scissors and one curved grasper.

- Cuschieri set, consisting of two instruments (one scissors and one grasper) proximally deviating curved coaxial instruments.

- Carus set, consisting of two instruments (one scissors and one grasper) with a curved shape with a relatively big radius starting at the middle of the shaft and ending exactly in the virtual line between instrument grip and tip.
- Leroy set, consisting of two instruments (one scissors and one grasper) with double bending.

\section{Assessment}

At the end of each procedure, the performance of the access device was assessed based on objective (time to complete insertion) and subjective parameters (ie, significant leaking, movement constraint). Complications related to insertion were also recorded.

A performance assessment for the four sets of instruments was performed based on both objective (ie, overall procedure time; time to manage the pedicle; time to completely free the kidney) and subjective (ie, entry/exit of the instruments; triangulation; dissection up/down; dissection lateral; retraction; interdependence) parameters. The assessment tool used for subjective parameters was a Likert-type scale $(1=$ easy; $2=$ slightly difficult; $3=$ difficult; $4=$ very difficult; $5=$ prohibitive).

\section{Results}

Time needed for port insertion and starting $\mathrm{CO}_{2}$ insufflation was less than 1 minute in all the cases. Surgeons reported significant insufflant leakage in $58 \%$ of the cases. Significant movement constraint was reported in $50 \%$ of the cases. There were no complications related to port insertion. The X-CONE seal allowed use of the different instruments with variable configuration.

Nephrectomy was successfully completed in 10/12 (83\%) cases. Mean operative time was $8.3 \pm 4.2$ minutes, being lower for the Carus group $(4.5 \mathrm{~min})$ and higher for the standard group (13 min). Mean time to manage the renal pedicle was $4.2 \pm 3.1$ minutes. There was one complication (bowel injury

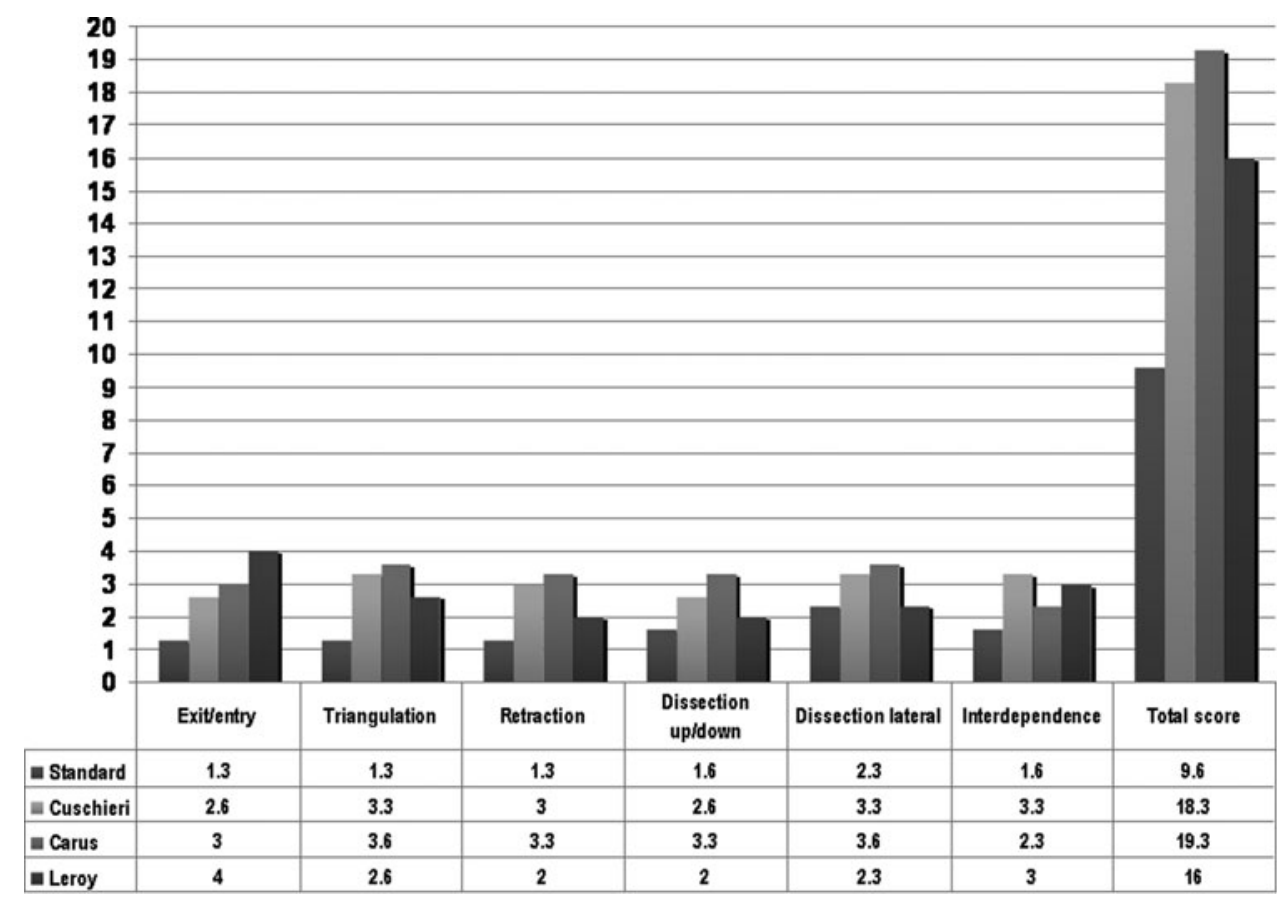

FIG. 3. Performance assessment of prebent instruments for laparoendoscopic single-site nephrectomy (values expressed as means on a scale 1 to $5: 1=$ easy; $2=$ slightly difficult; $3=$ difficult; $4=$ very difficult; $5=$ prohibitive; total score ranging $6-30$ ). 

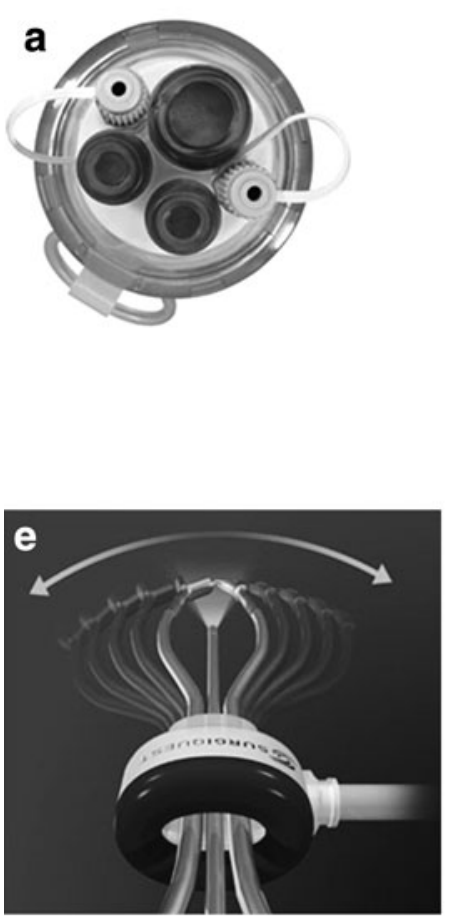

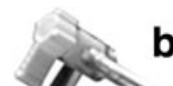

b
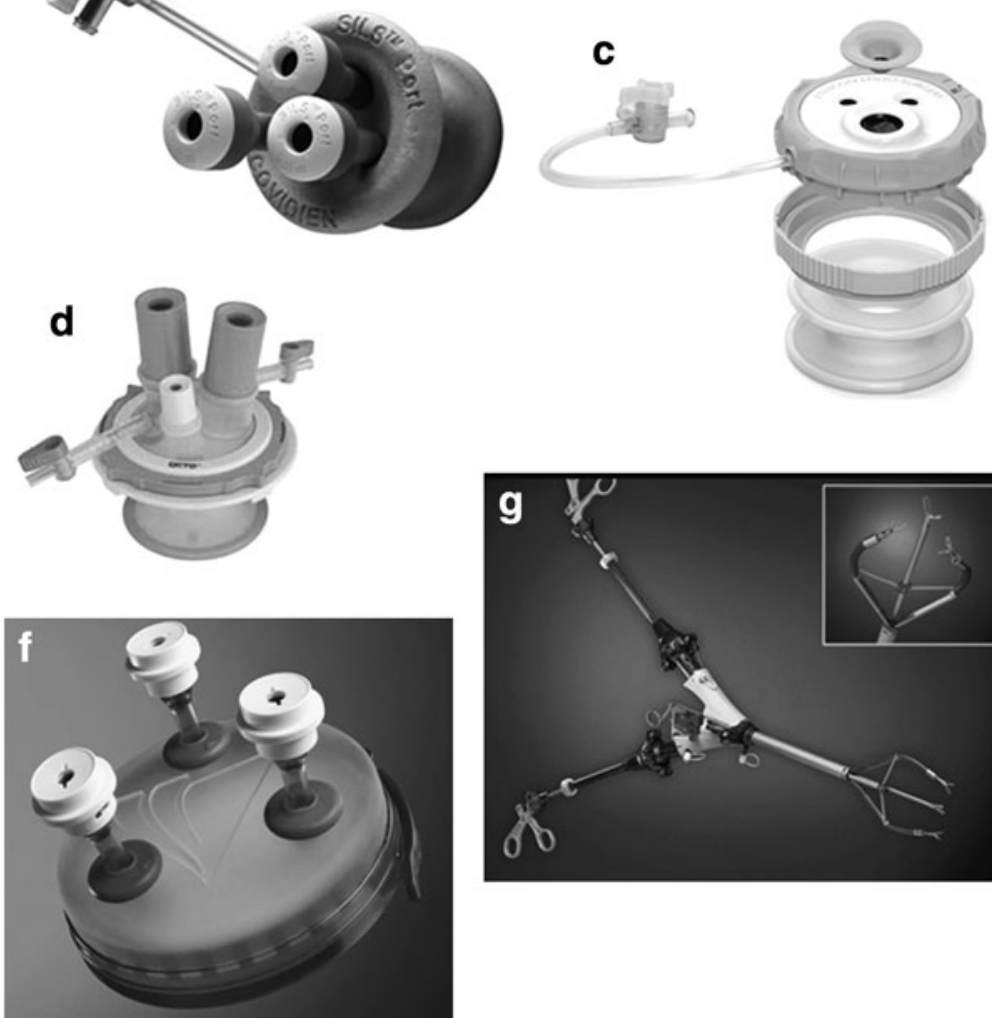

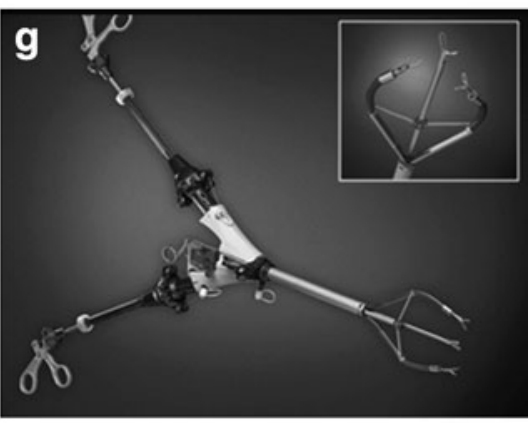

FIG. 4. Currently available platforms for single-port surgery: (a) TriPort, ${ }^{\mathrm{TM}}$ Olympus; (b) SILSTM Port, Covidien; (c) SSL Access System, Ethicon; (d) OCTO ${ }^{\mathrm{TM}}$ Port, Dalimsurg; (e) AirSeal, ${ }^{\mathrm{TM}}$ SurgiQuest; (f) GelPOINT, ${ }^{\circledR}$ Applied Medical; (g) SPIDER, TM TransEnterix.

during instrument insertion). Significant external clashing was noted in $41 \%$ of cases.

Scores for each assessed parameter are summarized in Figure 3.

\section{Discussion}

LESS surgery aims to mimic standard laparoscopic techniques but with the potential advantages of minimizing skin incision morbidity and providing superior cosmesis. ${ }^{1}$

When a single-port technique for LESS surgery is applied, a multilumen port is needed to ensure multiple instrument insertion. In this regard, several options have become available in the last few years and proved to be effective (Fig. 4). ${ }^{5,6}$ Nevertheless, although specific-cost analyses have not been reported, a recognized issue is cost of single-port access platforms. To address this, homemade devices have been created, but until now, no reusable commercially available single-port platform had been described. ${ }^{7}$

The reusable platform described in the present study represents an interesting low-cost option for LESS surgery. Its simple design allowed a quick intra-abdominal positioning through a minilaparotomy, with virtually no need for a learning curve, because the two halves of the device acted similarly to retractors. No complications were related to the port insertion. Of note, two problems were reported by the surgeons in $50 \%$ or more of the cases: Insufflant leakage, which was resolved by using a tourniquet, and significant movement constraint related to the rigid profile of the platform. The skin and subcutaneous tissue of the pig do not possess as much tensile strength as human tissue, and this aspect may explain some of the insufflant leakage around the X-CONE in the animal model. All surgeons did note that the X-CONE seal allowed use of the different instruments with a well-designed variable configuration.

The performance of LESS procedures is associated with various technical challenges, including minimal or no triangulation when using conventional laparoscopic instruments, leading to internal and external clashing and poor range of motion. ${ }^{8}$

To overcome these limitations, various disposable instruments have been developed. ${ }^{5,6}$ Real Hand ${ }^{\mathrm{TM}}$ (Novare Surgical, Cupertino, CA) and the Autonomy Laparo-Angle ${ }^{\mathrm{TM}}$ series (Cambridge Endo, Framingham, MA) are articulating instruments that are designed to provide triangulation and external spacing for laparoscopic and LESS procedures. A less expensive alternative is the Roticulator ${ }^{\mathrm{TM}}$ series (Covidien, Mansfield, MA) that was designed originally for standard laparoscopic surgery. Despite providing some triangulation, this technology only provides articulation in one plane, and thus the degrees of freedom are necessarily decreased.

The costs of single-port devices and disposable articulating instruments limit the availability of this technology signifi- 
cantly. Cost differences between single- and multiple-port laparoscopy are being studied but are not yet known. Each disposable single-port device costs approximately $\$ 400$ and disposable articulating instruments $\$ 1000$ to $\$ 2000$. Reusable access device and instruments described in this study have been very recently launched in the US market with an estimated cost of $\$ 2000$ for the X-CONE and $\$ 1000$ for any prebent instrument.

Surgeons who perform LESS surgery are also using traditional rigid, straight instruments, claiming that articulating instruments are not strictly necessary. Branco and coworkers ${ }^{9}$ evaluated umbilical LESS urologic surgery using conventional laparoscopic instruments and ports. They performed several procedures, including nephrectomies, adrenalectomy, ureterolithotomy, and retroperitoneal mass resection, and concluded that it is feasible and safe to use conventional laparoscopic instruments.

Flexible instruments do not overcome the problem of instrument crossing at the point of entry into the multichannel port, and the force applied to the instrument dissipates along the flexible portion of the shaft, limiting maneuverability during dissection.

Prebent instruments have been introduced with the aim of minimizing instrument clashing outside the port, providing triangulation in the operative field and better force distribution during dissection. ${ }^{10}$ They may be more cost-effective, because they are reusable compared with disposable articulating instruments.

Stolzenburg and associates ${ }^{11}$ recently performed a comparative evaluation in dry laboratory and porcine model nephrectomies among conventional, flexible, and prebent instruments in an attempt to elucidate instrument effectiveness and maneuverability. Prebent instruments proved to have better maneuverability and allowed shorter operative times.

In the present study, we compared four different recently introduced sets of prebent instruments in the porcine model based on time requirement to complete a nephrectomy and maneuverability of instruments as judged by three experienced surgeons. These sets of instruments had been designed and marketed for gallbladder removal.

All the sets reported a score between 2 (slightly difficult) and 3 (difficult) in all parameters subjectively assessed by the surgeons performing LESS nephrectomy. Of note, the standard set, consisting of a straight scissors and a curved grasper, was found to be the easiest to use based on all parameters reported.

Prebent instruments have the potential advantage of causing minimal interference with other instruments in the operative field as well as limiting clashing outside of the port. Nevertheless, in this experience, external clashing was noted in five cases ( $41 \%$ of the procedures) while only one occurred with the standard set.

The use of an extra-long laparoscope can further assist in preventing clashing of the camera with the instruments. The extra length separates the camera head and light cord from the area that is occupied by the surgeon's hands. The scopes used in this study also accept a right-angle light cord adapter that brings the light cord in line with the camera cord, further reducing interference with the surgeon.

An important limitation of our experimental study was the limited sample size. Moreover, because expert surgeons performed all procedures, difficulties in the manipulation of instruments may have been overcome by their level of expertise.

It is expected that newly introduced instruments could be further evaluated in other animal experimental procedures as well as clinical investigations to draw scientifically solid conclusions for their advantages. Clinical evaluation may be the most appropriate way to definitively assess the efficacy of new platforms and prebent instruments. None of the authors has had any direct clinical experience with the $\mathrm{X}$-Cone. Thus, further assessment in a clinical setting is expected. So far, clinical application of the device has not been reported in the literature. To our knowledge, the device has been anecdotally used by urologists in Europe, and it has been approved recently by the Food and Drug Administration and launched in the US market. Regarding the issue of cost-effectiveness, it is wise to state that, with reusable instruments, durability and reliability play an important role. This was not assessed in the study, however.

\section{Conclusions}

The $\mathrm{X}$-CONE is a reusable access device for single-port surgery that allows surgeons to have easy access to the intraperitoneal cavity to perform LESS surgery. The reusable properties of this platform can provide cost savings compared with other disposable devices. Further clinical assessment is warranted.

Prebent instruments represent attractive low-cost alternatives for LESS instrumentation. In the porcine model, the standard set allowed surgeons to perform LESS nephrectomies with less difficulty compared with other sets of prebent instruments.

\section{Disclosure Statement}

No competing financial interests exist.

\section{References}

1. Gill IS, Advincula AP, Aron M, et al. Consensus statement of the consortium for laparoendoscopic single-site surgery. Surg Endosc 2010;24:762-768.

2. Rané A, Rao P, Rao P. Single-port access nephrectomy and other laparoscopic urologic procedures using a novel laparoscopic port (R-Port). Urology 2008;72:260-264.

3. Raman JD, Bensalah K, Bagrodia A, et al. Laboratory and clinical development of single keyhole umbilical nephrectomy. Urology 2007;70:1039-1042.

4. White WM, Haber GP, Goel RK, et al. Single-port urological surgery: Single-center experience with the first 100 cases. Urology 2009;74:801-804.

5. Autorino R, Stein RJ, Lima E, et al. Current status and future perspectives in laparoendoscopic single-site and natural orifice transluminal endoscopic urological surgery. Int J Urol 2010;17:410-431.

6. Kommu S, Rané A. Devices for laparoendoscopic singlesite surgery in urology. Expert Rev Med Devices 2009;6: 95-103.

7. Tai HC, Lin CD, Wu CC, et al. Homemade transumbilical port: An alternative access for laparoendoscopic single-site surgery (LESS). Surg Endosc 2010;24:705-708. 
8. Canes D, Desai MM, Aron M, et al. Transumbilical singleport surgery: Evolution and current status. Eur Urol 2008; 54:1020-1029.

9. Branco AW, Kondo W, Stunitz LC, et AL. Transumbilical laparoscopic urological surgery: Are special devices strictly necessary? BJU Int 2009;104:1136-1142.

10. Raman JD, Cadeddu JA, Rao P, Rane A. Single-incision laparoscopic surgery: Initial urological experience and comparison with natural-orifice transluminal endoscopic surgery. BJU Int 2008;101:1493-1496.

11. Stolzenburg JU, Kallidonis P, Oh MA, et al. Comparative assessment of laparoscopic single-site surgery instruments to conventional laparoscopic in laboratory setting. J Endourol 2010;24:239-245.
Address correspondence to: Riccardo Autorino, M.D., Ph.D. Glickman Urological and Kidney Institute Cleveland Clinic 9500 Euclid Avenue Q10 Cleveland, $\mathrm{OH} 44194$

E-mail: autorir@ccf.org ricautor@tin.it

$\begin{aligned} & \text { Abbreviations Used } \\ & \text { LESS }=\text { laparoendoscopic single-site } \\ & \mathrm{US}=\text { United States }\end{aligned}$

